\title{
Optimal anticipative congestion control of flows with time-varying input stream ${ }^{\text {भ }}$
}

\author{
Peter Jacko ${ }^{\mathrm{a}, *}$, Brunilde Sansò ${ }^{\mathrm{b}}$ \\ a BCAM-Basque Center for Applied Mathematics, Bilbao, Spain \\ ${ }^{\mathrm{b}}$ GERAD and École Polytechnique de Montréal, Montréal (Québec), Canada
}

\section{A R T I C L E I N F O}

\section{Article history:}

Received 7 October 2010

Received in revised form 22 July 2011

Accepted 28 October 2011

Available online $\mathrm{xxxx}$

\section{Keywords:}

Markov decision process

Threshold policy

Restless bandits

Whittle index

Congestion control

Active queue management

Fairness

Transmission Control Protocol (TCP)

Explicit Congestion Notification (ECN)

\begin{abstract}
A B S T R A C T
This paper is concerned with a new type of congestion control method that we call anticipative congestion control, which exploits probabilistic information available at a network node about congestion at other nodes. Motivated by the Internet flows behaving according to the Transmission Control Protocol, we consider a flow with time-varying input stream. We design a Markov decision process model for flow admission control and characterize the Whittle index in a closed form. This index measures the efficiency of flow data transmission at a router. We prove that such an index policy is optimal and that it further implies optimality of threshold policies. We apply the results to obtain an expression of the index for a single-bottleneck flow under several types of fairness criteria.
\end{abstract} (C) 2011 Elsevier B.V. All rights reserved.

\section{Introduction}

This paper deals with what we call anticipative congestion control which is a method whereby the router that has to take a decision to control congestion, such as dropping/marking a flow/packet, does it based not only on its own congestion information, but also on the probability that the accepted flow/packet will be dropped downstream. As Floyd and Fall [1] pointed out, traffic lacking end-to-end congestion control may cause congested links sending packets that will only be dropped later in the network. Since dropping a packet on its route implies that all the scarce resources it has consumed so far are wasted, such an anticipative congestion control may be highly valuable for the network performance in periods of congestion. It is then intuitively appealing that when a scarce resource is to be allocated to a packet, the possibility of getting that packet lost in the remainder of its route should be taken into account. A first important assumption of this work is, therefore, that routers have probabilistic information about actual congestion downstream. Another assumption of our proposal is that the method is applied to Internet flows behaving according or similar to the Transmission Control Protocol

\footnotetext{
败 This research has been supported in part by the Spanish Ministry of Education and Science under grant MTM2004-02334 and an associated Postgraduate Research Fellowship funded by the European Social Fund, by the Autonomous Community of Madrid-UC3M through grant UC3M-MTM-05-075, by the European Union's Network of Excellence Euro-NGI, by grant MTM2010-17405 (Ministerio de Ciencia e Innovación, Spain), grant PI2010-2 (Department of Education and Research, Basque Government), and by Canada's National Science and Engineering grants. This work was mostly done during the stay of P. Jacko at GERAD, Canada, in 2007. Earlier versions of this work appeared in $[30,18]$. The authors are grateful for discussions with Urtzi Ayesta and Konstantin Avrachenkov that have led to improvements in the presentation of the ideas in the paper.

* Corresponding author. Tel.: +34 946567 842; fax: +34 946567843.

E-mail addresses: jacko@bcamath.org, peter.jacko@gmail.com (P. Jacko), brunilde.sanso@polymtl.ca (B. Sansò).
} 


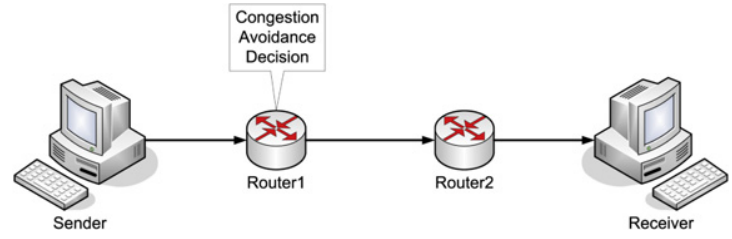

Fig. 1. A design of an end-to-end connection.

(TCP). The distinguishing feature of TCP is the time-varying input stream (sending rate), which is being deterministically and dynamically modified by the sender reacting to the congestion signals received from the network. However, such congestion signals (that include dropped or marked packets) are perceived as stochastic, since they depend on the activity of other users and on the algorithms implemented at network routers.

We build on an improved idea of Wischik [2], who concluded that a fair dropping/marking of a flow should reflect (i) how much of the capacity it uses, and (ii) the congestion state at the router. We extend the latter to (ii') the congestion state at the router and the remainder of the route. More information at network nodes may result in a more efficient resource allocation. Notice that the information about network congestion gathered by intermediate nodes has a significantly lower propagation delay than the one that end-users obtain from packet acknowledgments. Similarly, the delay in the response to congestion is also lower for nodes than for users. The recently proposed explicit congestion notification (ECN) mechanism may not only decrease packet losses directly by marking instead of dropping packets, but may also provide useful information for a more efficient resource allocation at network nodes.

To illustrate the idea on a simple example, consider an end-to-end connection that includes two bottleneck routers, as in Fig. 1. If Router2 is busy (yet still has some free capacity in the buffer) and Router1 is able to anticipate it, then congestion notification decisions at Router 1 should take into account the input rate of an incoming flow. If the rate is small, so that Router2 would be able to transmit the arriving data, then they should be transmitted at Router1. On the other hand, if the input rate is too high, so that Router2 is very likely to drop it, the flow should be dropped already at Router1; or, it could be a strong candidate for getting a congestion signal from the congestion notification mechanism implemented at Router 1.

The data of the flow generate certain reward for its receiver, if they are delivered, which can be achieved only if they are transmitted by the router. The difficulty is that these flows are dynamically changing their input rate, so the rewards may increase or decrease over time. Thus, the question is whether to exploit the present rewards by transmitting at the input rate, or to take a myopically suboptimal action which may yield higher rewards further downstream or to the following packets arriving at the router.

The objective of this paper is to formalize such an intuition. We deal with typical concave utility functions, which were summarized as $\alpha$-fairness in [3] and generalized in [4]. For particular values of $\alpha, \alpha$-fairness covers throughput maximization, proportionally fair assignment, delay minimization, and approximates max-min fairness arbitrarily closely. We let the router calculate the $\alpha$-fair price of the entire flow based on the input rate and the current congestion information and compare it to a transmission cost given as a parameter. Such a price is defined by the Whittle index [5] in this paper.

In contrast to the anticipative congestion control proposed here, the mechanisms implemented so far are either reactive or preventive. Reactive congestion control such as the buffer tail dropping policy showed to be prone to creating various serious problems in the networks (cf. [6]) and to have a significant negative impact on the efficiency of scarce resources (bandwidth and buffer space) allocation in networks. Alternative proposals focused on preventive congestion control developing active queue management mechanisms, such as RED [7], BLUE [8], and a palette of their variants, which try to detect local congestion in its early stage and warn the traffic sources expecting that they decrease their input rates. Recently, ECN has become increasingly deployed in the Internet. Explicitly marked packets should be understood by users as a warning about possible dropping of packets in a very near future. However, such a preventive schemes are heavily sensitive to several parameters and flow types, and there are no general rules for their proper tuning even after two decades of research.

Our approach differs from the preventive congestion control mainly by trying to exploit available information about congestion at other network nodes, not only about the congestion at the node where this control is implemented. Gathering of such information inside the network may be costly, however, increasing ECN deployment improves the possibilities for routers to have fresh news from other network nodes. Similar assumptions about information at network nodes were considered for congestion control problems in [9-11]. They showed that communication across network nodes, especially the neighboring ones, is beneficial and the latter two discussed also practical implementation of such information gathering.

\subsection{General Markov decision process model}

We model the anticipative congestion control problem in the framework of Markov decision processes (MDPs). In particular, we present a binary-action MDP (i.e., a restless bandit) model for the router-based control of the flow. Consider the time slotted into discrete time epochs $t \in \mathcal{T}:=\{0,1,2, \ldots\}$. The period between two epochs is fixed and approximates the flow's round-trip time (RTT), so it can be defined by the propagation RTT, while the congestion delays (i.e., the time spent in the router buffers) are ignored. We assume that all packets are of the same size, which we further define to be one 
bandwidth/buffer capacity unit (this restriction could be dropped with the cost of a more complicated notation, since one would need to have the system parameters both in packet units and in bits). We define the following parameters of the system:

- the state space $\mathcal{N}:=\{0,1, \ldots, N-1\}, N \geq 1$, is a set of possible levels of the input stream;

- the workload $W_{n}^{\text {sent }}$ (in number of packets) sent by the sender of the flow at state $n$;

- the action space $\mathcal{A}:=\{0,1\} ; A:=2$ is the number of available actions by the router for the flow;

- the one-period transition probability matrix $\boldsymbol{P}^{a}$ for the flow states if action $a$ is applied at the router;

- the one-period expected workload $W_{n}^{a}$, or bandwidth used, of the flow at state $n$ if action $a$ is applied at the router;

- the one-period expected reward $R_{n}^{a}$, or fairness, obtained of the flow at state $n$ if action $a$ is applied at the router.

Suppose that the flow starts in an initial state $i \in \mathcal{N}$ and that the end-users' impatience follows a geometric distribution with mean $1 /(1-\beta)$. This may well be due to an impatience of the sender, of the receiver, or due to external factors such as broken connections (see, e.g., [12]). Thus, the sender has packets available for transmission during a random number of periods, given by the probability $0<1-\beta<1$ that the flow is terminated before the next time epoch. Except for the end-users' impatience, the flow is considered persistent.

Denote by $X(\cdot)$ the state process of the flow and by $a(\cdot)$ the action process by the router at epochs $t \in \mathcal{T}$. The system dynamics is as follows (see Fig. 1). At epoch $t$, the sender finds herself at its state $X(t) \in \mathcal{N}$ and sends the workload of $W_{X(t)}^{\text {sent }}$ packets to the router. The router observes the flow state $X(t)$, decides the flow action $a(t) \in \mathcal{A}$, and instantaneously transmits $0 \leq W_{X(t)}^{a(t)} \leq W_{X(t)}^{\text {sent }}$ packets. The transmitted packets with possible losses (at subsequent routers further downstream) arrive to the receiver, who obtains the reward $R_{X(t)}^{a(t)}$. If the router transmitted the packets of the flow without any congestion warning and if there was no congestion downstream, then the receiver sends complete acknowledgments back to the sender; otherwise only partial acknowledgments are sent. Next, with probability $1-\beta$ the flow terminates so that there are no more packets sent by the sender in the future time epochs and no more rewards earned by the receiver. If not terminated, then the sender's next-epoch state $X(t+1)$ is set according to $\boldsymbol{P}^{a(t)}$ and the process is repeated. The transition probability matrix $\boldsymbol{P}^{a(t)}$ must thus capture whether the workload was received without congestion, which is given by the complete acknowledgments of the receiver sent back to the sender. We emphasize that the sender makes no decisions and the input stream dynamics is Markovian. The decision upon $a(t)$ is taken by the router.

Let $v$ be the per-packet transmission cost considered as a parameter of the router in which the control is to be implemented. Let $\Pi$ be the set of all history-dependent randomized policies for the flow. Then our objective is to find a policy $\pi^{*} \in \Pi$ resolving the following $\nu$-parameter problem:

$$
\max _{\pi \in \Pi} \mathbb{E}_{i}^{\pi}\left[\sum_{t=0}^{\infty} \beta^{t}\left(R_{X(t)}^{a(t)}-v W_{X(t)}^{a(t)}\right)\right],
$$

where $\mathbb{E}_{i}^{\pi}$ denotes the expectation over the state process $X(\cdot)$ and over the action process $a(\cdot)$, conditioned on the initial state $X(0)=i$ and on policy $\pi$.

\subsection{Related models}

Well-studied in the queueing theory literature is the admission control problem, in which a decision maker can choose between two static input rates, typically corresponding to admission and rejection of the flow. This can be seen as a bi-criteria optimization problem resolving the trade-off between earning a reward from accepting the flow or paying a rejection cost, pioneered by Naor [13] for Poisson arrivals. For the same arrival (input) process, Niño-Mora [14] identified an optimal index policy, using the Whittle index. However, the TCP-like behavior described in the previous section does not result in a Poisson process for the number of arriving packets, so these models are inadequate for studying flows in the Internet that typically dynamically change their input stream. Piunovskiy [15] introduced a parametric model with controlled input stream that can be chosen from a fixed interval of rates and proved optimality of a bang-bang control depending on the congestion (queue length) at the router. Again, such a policy is in a big contrast with the Internet flows behaving according to TCP.

Multi-flow congestion control at a router can be decomposed into a collection of per-flow admission control problems. Such observations were made in $[16,10]$, who studied stability of the problem using a fluid-flow model. They illustrated that properly designed admission control can be shown to be sufficient for both an efficient congestion control and an efficient routing. Building on an analogy with the multi-armed restless bandit problem introduced in [5], a Lagrangian decomposition into parametric admission control subproblems was designed in [17] for a buffer sharing problem with Poisson arrivals of new packets. Jacko [18, Chapter 6] developed within the same framework an analogous decomposition for flows considered in this paper. Thus, it is enough to focus here on control problem of an individual flow in isolation, captured in (1).

Closely related to our paper is a wide stream of literature on network economics and pricing (see, e.g., [19]) aiming at improving network resource allocation. The book [20] and the surveys [21-24] provide summaries of the most important mathematical models and results on congestion control for whole networks. The typical objective is to optimize the sum of user's utility functions, and is approached by showing that such a problem can be decomposed into per-user problems of setting input rates in an adaptive (reactive to network congestion signals) manner. The typical result is that a particular 
type of fairness is achieved if all the users have utility functions of same type. The flows are assumed to be persistent and they are studied in the fluid limit regime.

\subsection{Goals and contributions}

By anticipative congestion control problem we mean to implement particular control methods using the actual network congestion information to reduce overall congestion for the route. In this paper we show how several types of fairness could be achieved by introducing the transmission index to evaluate the efficiency of flow data transmission as a function of its current input rate and current network congestion state.

There is an interesting consequence of taking the network congestion information into account on fairness. Our results imply that two flows arriving at the same rate should not be treated equally if we know that one of them is routed to a congested link and the other one to a congestion-free link. In other words, flows are treated fairly according to what the network can transmit, and not according to what the flows want to transmit as it is usually assumed in the existing concepts of fairness.

In Section 2 we present an MDP (restless bandit) model of anticipative congestion control of an individual flow. The flow dynamics and the parameters are defined in more detail there. We show that the decisions upon dropping and marking of flow packets can be transformed into a flow admission control problem.

In Section 3 we employ the restless bandits methodology surveyed in [25] to obtain an optimal solution for the anticipative congestion control of an individual flow via the Whittle (marginal productivity) index. This index captures the value of network services to users, so it can be interpreted as a transmission index in our context. It can be seen as a list of certain internal prices, and can be implemented in congestion notification mechanisms in order to resolve the fairness problem between different flows.

As an example of applicability of our general results, Section 4 deals with admission control in a tail dropping router of a single-bottleneck network under different fairness criteria known as generalized $\alpha$-fairness. The extension to a multibottleneck network is illustrated by a numerical example in Section 5 .

\section{Anticipative congestion control problem}

In this section we consider an individual flow requiring router resources (buffer space and bandwidth capacity). We present an MDP model for flows that behave under the any-increase/restart dynamics, henceforth called the restarting flow. This approximately resembles the dynamics of several TCP variants, including TAHOE, CUBIC and COMPOUND. A similar model could be developed for other flow dynamics, but this has been left out of the scope of this paper.

We assume that the router has two available actions to choose from. One of these actions is transmitting the packets of the flow without warning. The other action is sending a congestion warning, which, depending on the router variant, may mean: (i) blocking the flow for a tail dropping router, (ii) dropping some packets of the flow for a router with an active queue management mechanism (e.g., RED), or (iii) marking the flow for an ECN router.

In order to define the problem of anticipative congestion control of an individual flow, we will use information about the router variant (to know what to decide upon), the current flow input stream (to know the flow's bandwidth requirements), and the probability of losses downstream the flow's route (to be anticipative). In the following subsections we present a formal model of the restarting flow. An optimal anticipative congestion control is derived in Section 3.

\subsection{Markov decision process model of restarting flow}

The restarting flow in every period increases its sending rate defined by the actualWindow variable by a discrete increment up to a certain maximumWindow constant unless the flow sender is warned about congestion, when it restarts by setting the sending rate to a certain minimumWindow constant. We assume that the constants $0<$ minimumWindow $\leq$ maximumWindow are given in advance, as they are typically set by the users' operating systems.

The states $n \in \mathcal{N}$ denote possible levels of the sending rate, i.e., of the actualWindow variable. The 0-th state represents actualWindow $=$ minimumWindow, and the $(N-1)$-th state represents actualWindow $=$ maximumWindow. The actualWindow variable in state $n$ assumes the value $W_{n}^{\text {sent }}$ (in packets/RTT), which can therefore be interpreted as the bandwidth capacity the flow requires for complete data transmission at the current period. Hence, in the following we assume that $0<W_{0}^{\text {sent }}:=$ minimumWindow $<W_{1}^{\text {sent }}<\cdots<W_{N-2}^{\text {sent }}<W_{N-1}^{\text {sent }}:=$ maximumWindow. The schematic behavior of the restarting flow as a Markov chain is shown in Fig. 2, where "OK" represents a congestion-free reception of the flow data by the receiver (complete acknowledgments) and "NO" represents a congestion-experienced transmission (incomplete acknowledgments).

Since no decisions are taken by the sender, congestion control decisions must be taken by the routers. At a particular router on the connection route we want to decide whether the incoming packets of the flow in state $n$ should be transmitted without any congestion warning (achieved by action $a(t)=1$ of transmitting $W_{n}^{1}:=W_{n}^{\text {sent }}$ packets), or warned by employing a congestion control function (action $a(t)=0$ of transmitting $0 \leq W_{n}^{0} \leq W_{n}^{\text {sent }}$ packets) depending on the router variant. Thus, the parameter $W_{n}^{0}$ gives us the flexibility to consider router variants with different congestion warning. 


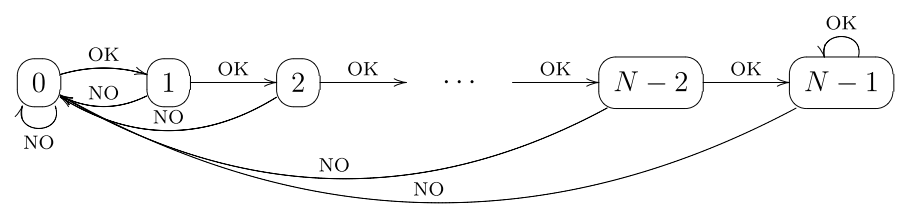

Fig. 2. A model of the restarting flow as a Markov chain. The arrows represent one-period transitions among the states $0,1, \ldots, N-1$ after a congestionfree (OK) and a congestion-experienced (NO) transmission.

If the flow is warned at the router, then the flow restarts to state 0 . If the packets of the flow are transmitted without warning, then with probability $0<p_{n} \leq 1$ of congestion-free transmission it increases the input rate from $W_{n}^{\text {sent }}$ to $W_{n+1}^{\text {sent }}$ (or remains at the maximumWindow rate if being in state $N-1$ ). On the other hand, the flow being in state $n$ restarts to state 0 with probability $1-p_{n}$, reflecting the probability of experiencing congestion in the remainder of its route. The assumption $p_{n} \neq 1$ is what makes our model suitable for the anticipative congestion control problem, whereas the single-bottleneck problem is recovered if $p_{n}=1$ for all $n \in \mathcal{N}$. Note that such a restarting behavior is similar to the well-known models with total catastrophes (see e.g., [26]).

Given the actions interpretation above, $R_{n}^{1}$ is the expected one-period reward (fairness) from a transmitted packets of the flow and $R_{n}^{0}$ is the expected one-period reward (fairness) from a warned flow. We will find it convenient to further decompose $R_{n}^{1}$ into the congestion-free reward $R_{n}^{1+}$ and the congestion-experienced reward $R_{n}^{1-}$, so that $R_{n}^{1}:=p_{n} R_{n}^{1+}+(1-$ $\left.p_{n}\right) R_{n}^{1-}$. Thus, $R_{n}^{0}$ and $R_{n}^{1}$ can capture the system's sensitivity to dropped or marked packets, and should also depend on $W_{n}^{0}$ and $W_{n}^{1}$, respectively. The difference of receiver rewards and workload transmission costs (i.e., $R_{n}^{0}-v W_{n}^{0}$ and $R_{n}^{1}-v W_{n}^{1}$ ) will be henceforth called the net reward under transmission cost $v$.

In summary, our MDP model for the router-based control of the restarting flow is defined as follows:

- State space is $\mathcal{N}:=\{0,1, \ldots, N-1\}$.

- Actions: active action (transmitting) and passive action (warning) are available in each state.

- Dynamics if active: If the flow is in state $n$ and the flow packets are transmitted at a given period, then during that period

- with probability $p_{n}>0$ : it generates net reward $R_{n}^{1+}-v W_{n}^{1}$ and the flow moves to state $n+1$ for the next period (or remains in $N-1$, if $n=N-1$ )

- with probability $1-p_{n}$ : it generates net reward $R_{n}^{1-}-v W_{n}^{1}$ and the flow moves to state 0 for the next period.

- Dynamics if passive: If the flow is in state $n$ and the flow is warned at a given period, then during that period it generates net reward $R_{n}^{0}-v W_{n}^{0}$ and the flow moves to state 0 for the next period.

\section{Optimal solution}

In this section we solve problem (1) using the restless bandit methodology surveyed in [25], whose central concept is that of problem's indexability. Recall that $v$ denotes the transmission cost (or, the cost of providing bandwidth capacity) paid for each unit of router's bandwidth required by the flow, and let us consider it as a parameter for the moment.

Definition 1 (Indexability). We say that problem (1) is indexable, if there exist real numbers $\left\{v_{n}, n \in \mathcal{N}\right\}$ such that the following holds for every state $n \in \mathcal{N}$ :

1. if $v_{n} \geq v$, then it is optimal to transmit in state $n$, and

2. if $v_{n} \leq v$, then it is optimal to warn in state $n$.

The function $n \mapsto v_{n}$ is called the index, and $v_{n}$ 's are called the index values.

Whittle [5] realized that restless bandit's indexability cannot be taken for granted and provided a non-indexable problem instance. The index is therefore often called the Whittle index. The ultimate goal of this section is thus to establish indexability of problem (1) and characterize the index values. We will rely on a sufficient indexability condition introduced and developed in $[27,14]$.

Equivalently, indexability means that the set of states in which it is optimal to transmit increases monotonically from an empty set to $\mathcal{N}$ as the transmission cost $v$ decreases from $+\infty$ to $-\infty$. In particular, this implies economically sound properties that if the transmission cost $v$ is high enough, then it cannot be optimal to transmit the data of the flow in any state, and if the transmission cost $v$ is low enough, then it must be optimal to transmit the data of the flow in any state. While there are several ways how the set of states in which it is optimal to transmit may monotonically increase, we identify in this paper conditions that imply optimality of threshold policies; other possible policy structures are ignored. We therefore propose to call the index in such a case the transmission index, as it identifies until which threshold state transmitting is optimal.

The following three subsections prepare the ground and reduce the notational and analytical complexity of the proof of the main results of this paper, which are presented in Section 3.5. 


\subsection{Performance region}

Consider now the flow control problem (1). Thus, we look for a policy maximizing the expected total net rewards under the $\beta$-discounted criterion. To evaluate a policy $\pi$ under the $\beta$-discounted criterion, we consider the following two measures. Let $\mathbb{W}_{i}^{\pi}:=\mathbb{E}_{i}^{\pi}\left[\sum_{t=0}^{\infty} \beta^{t} W_{X(t)}^{a(t)}\right]$ be the expected total $\beta$-discounted bandwidth utilization if starting from state $i$ under policy $\pi$. For convenience, we will also call $\mathbb{W}_{i}^{\pi}$ the expected total $\beta$-discounted work, since the bandwidth utilization can be seen as the work performed by the router in order to transmit the flow data. Analogously, we denote by $\mathbb{R}_{i}^{\pi}:=\mathbb{E}_{i}^{\pi}\left[\sum_{t=0}^{\infty} \beta^{t} R_{X(t)}^{a(t)}\right]$ the expected total $\beta$-discounted reward if starting from state $i$ under policy $\pi$.

The objective $(1)^{1}$ is for each transmission cost $v$,

$$
\max _{\pi \in \Pi} \mathbb{R}_{i}^{\pi}-v \mathbb{W}_{i}^{\pi} .
$$

\subsection{Normalized optimization problem}

Any restless bandit can be normalized so that the rewards are non-zero only under the active action and the two optimization problems' objective functions are equivalent (see, e.g., [14], p. 383). In a parameterized model this can be accomplished by defining the active-action reward and work via

$$
\boldsymbol{R}:=\boldsymbol{R}^{1}-\left(\boldsymbol{I}-\beta \boldsymbol{P}^{1}\right)\left(\boldsymbol{I}-\beta \boldsymbol{P}^{0}\right)^{-1} \boldsymbol{R}^{0}, \quad \boldsymbol{W}:=\boldsymbol{W}^{1}-\left(\boldsymbol{I}-\beta \boldsymbol{P}^{1}\right)\left(\boldsymbol{I}-\beta \boldsymbol{P}^{0}\right)^{-1} \boldsymbol{W}^{0} .
$$

This can be simplified for our model (see Appendix B) to

$$
R_{n}:=\left(R_{n}^{1}-R_{n}^{0}\right)+\beta p_{n}\left(R_{n+1}^{0}-R_{0}^{0}\right), \quad W_{n}:=\left(W_{n}^{1}-W_{n}^{0}\right)+\beta p_{n}\left(W_{n+1}^{0}-W_{0}^{0}\right),
$$

where we have defined $R_{N}^{0}:=R_{N-1}^{0}$ and $W_{N}^{0}:=W_{N-1}^{0}$.

Thus, the above-defined dynamics of the system is modified in the following way:

- Dynamics if active: If the flow is in state $n$ and the flow is warned at a given period, then during that period it generates net reward $R_{n}-v W_{n}$ and

- with probability $p_{n}>0$ : the flow moves to state $n+1$ for the next period (or remains in $N-1$, if it is already there).

- with probability $1-p_{n}$ : the flow moves to state 0 for the next period.

- Dynamics if passive: If the flow is in state $n$ and the flow is warned at a given period, then during that period it generates no net reward and the flow moves to state 0 for the next period.

\subsection{Reduction to stationary policies}

Since for MDPs with finite state space and finite action space there exists an optimal policy which is deterministic, stationary, and independent of the initial state, we focus only on such policies and represent them via active sets $\& \subseteq \mathcal{N}$. In other words, a policy $\&$ prescribes to be active (to transmit) in states in $\delta$ and passive (to warn) in states in $\mathcal{N} \backslash \delta$.

In the remainder of this section we consider the normalized optimization problem under the set of all active sets. The above-defined total bandwidth utilization (total work) $\mathbb{W}_{i}^{\pi}$ and total reward $\mathbb{R}_{i}^{\pi}$ can readily be denoted by $\mathbb{W}_{i}^{\delta}$ and $\mathbb{R}_{i}^{\delta}$, respectively. Moreover, they satisfy the following balance equations, to be used later in the analysis.

Proposition 1. For any state $n$ and any active set $\&$ we have:

If state $n \in s$ and $n \neq N-1$, then

$$
\mathbb{R}_{n}^{\S}=R_{n}+\beta\left(p_{n} \mathbb{R}_{n+1}^{\delta}+\left(1-p_{n}\right) \mathbb{R}_{0}^{\S}\right), \quad \mathbb{W}_{n}^{\S}=W_{n}+\beta\left(p_{n} \mathbb{W}_{n+1}^{\S}+\left(1-p_{n}\right) \mathbb{W}_{0}^{\S}\right) .
$$

If state $N-1 \in \S$, then

$$
\mathbb{R}_{N-1}^{\&}=\frac{R_{N-1}+\beta\left(1-p_{N-1}\right) \mathbb{R}_{0}^{8}}{1-\beta p_{N-1}}, \quad \mathbb{W}_{N-1}^{8}=\frac{W_{N-1}+\beta\left(1-p_{N-1}\right) \mathbb{W}_{0}^{8}}{1-\beta p_{N-1}} .
$$

If state $n \notin$ s, then $\mathbb{R}_{n}^{\S}=\beta \mathbb{R}_{0}^{\S}, \mathbb{W}_{n}^{\S}=\beta \mathbb{W}_{0}^{\S}$. If state $0 \notin \S$, then $\mathbb{R}_{0}^{\S}=0, \mathbb{W}_{0}^{\S}=0$.

\footnotetext{
${ }^{1}$ Similarly could be formulated the problem under the time-average criterion. The index under the latter is obtained in the limit $\beta \rightarrow 1 \mathrm{from}$ the index under the discounted criterion [14].
} 


\subsection{Marginal reward and marginal work}

In this subsection we define measures of marginal reward and marginal work, and present some auxiliary results. Let $\langle a, \diamond\rangle$ be the policy that implements action $a$ in the initial period and policy $\&$ proceeds. For any state $n$ and policy $\&$, we consider the marginal reward defined as $r_{n}^{\S}:=\mathbb{R}_{n}^{\langle 1,8\rangle}-\mathbb{R}_{n}^{\langle 0,8\rangle}$, and the marginal work defined as $w_{n}^{8}:=\mathbb{W}_{n}^{\langle 1,8\rangle}-\mathbb{W}_{n}^{\langle 0,8\rangle}$. Finally, the marginal rate is denoted by $v_{n}^{8}:=r_{n}^{8} / w_{n}^{8}$. The following results are analogous to the balance equations in Proposition 1 .

Lemma 1. For any state $n \neq N-1$ and any active set $s$ we have

$$
\begin{array}{ll}
\mathbb{R}_{n}^{\langle 1,8\rangle}=R_{n}+\beta\left(p_{n} \mathbb{R}_{n+1}^{\&}+\left(1-p_{n}\right) \mathbb{R}_{0}^{\S}\right), & \mathbb{R}_{n}^{\langle 0,8\rangle}=\beta \mathbb{R}_{0}^{\&}, \\
\mathbb{R}_{N-1}^{\langle 1,8\rangle}=R_{N-1}+\beta\left(p_{N-1} \mathbb{R}_{N-1}^{\&}+\left(1-p_{N-1}\right) \mathbb{R}_{0}^{\&}\right), & \mathbb{R}_{N-1}^{\langle 0,8\rangle}=\beta \mathbb{R}_{0}^{\&}, \\
\mathbb{W}_{n}^{\langle 1,8\rangle}=W_{n}+\beta\left(p_{n} \mathbb{W}_{n+1}^{\&}+\left(1-p_{n}\right) \mathbb{W}_{0}^{\S}\right), & \mathbb{W}_{n}^{\langle 0,8\rangle}=\beta \mathbb{W}_{0}^{\S}, \\
\mathbb{W}_{N-1}^{\langle 1,8\rangle}=W_{N-1}+\beta\left(p_{N-1} \mathbb{W}_{N-1}^{\&}+\left(1-p_{N-1}\right) \mathbb{W}_{0}^{\&}\right), & \mathbb{W}_{N-1}^{\langle 0,8\rangle}=\beta \mathbb{W}_{0}^{\&} .
\end{array}
$$

Using the above lemma and the definition of the marginal reward and marginal work, respectively, we obtain the following identities.

Proposition 2. For any state $n \neq N-1$ and any active set $s$ we have

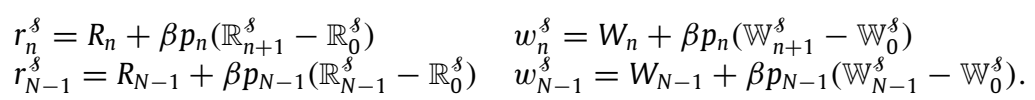

Finally, the next result is obtained by employing Proposition 1 into Proposition 2.

Proposition 3. If state $n \in \S$, then $r_{n}^{\S}=\mathbb{R}_{n}^{\S}-\beta \mathbb{R}_{0}^{\&}$ and $w_{n}^{\S}=\mathbb{W}_{n}^{\&}-\beta \mathbb{W}_{0}^{\&}$.

In order to characterize marginal work and marginal reward under a given active set $\$$, the above propositions allow us to narrow the focus to quantities $\mathbb{R}_{n}^{\delta}$ and $\mathbb{W}_{n}^{\delta}$ for $n \in \delta$ that can be obtained from the recursion in Proposition 1 , as we will see in the following subsection.

\subsection{Indexability and optimality of threshold policies}

Now we prepare the ground for establishing the structure of the optimal active sets (policies), under the following two conditions. First, we will use a natural monotonicity assumption upon congestion probabilities, termed deteriorating QoS (Quality of service). Indeed, one expects the network to increment losses as the required bandwidth per connection is increased.

Assumption 1 (Deteriorating QoS). The one-period work $W_{n}>0$ for all $n \in \mathcal{N}$, and $\frac{W_{n-1}}{p_{n-1}} \leq \frac{W_{n}}{p_{n}}$ for all $n \in \mathcal{N} \backslash\{0\}$.

Further, we will concentrate on the case, in which the one-period reward $R_{n}$ possesses a sort of concavity in $W_{n}$. The concavity behavior is a natural one for the fairness utilities in communications networks.

Assumption 2 (Concave Adjusted Reward). There is a real-valued function $R$ with $R(0) \geq 0$, which is concave on the domain $\left\{0, W_{0} / p_{0}, \ldots, W_{N-1} / p_{N-1}\right\}$, such that $R_{n} / p_{n}=R\left(W_{n} / p_{n}\right)$.

We will prove that, under these two conditions, the optimal policy for any transmission cost $v$ is a threshold policy belonging to family

$$
\mathcal{F}:=\left\{\mathcal{N}_{k-1}: k \in \mathcal{N} \cup\{N\}\right\}, \quad \text { where } \mathcal{N}_{k-1}:=\{0,1, \ldots, k-1\} .
$$

That is, for any transmission cost $v$ there is a threshold state $k$ such that it is optimal to transmit the packets of the flow if and only if it is in any state smaller than $k$. We will accomplish this by establishing PCL-indexability, introduced in $[27,14]$, of our problem with respect to the family of active sets $\mathcal{F}$ defined in (4).

Definition 2. Problem (2) is called PCL-indexable with respect to the family of active sets $\mathcal{F}$ defined in (4), if the following two conditions hold:

1. The marginal work $w_{n}^{\mathcal{N}_{k-1}}>0$ for all $n \in \mathcal{N}$ and $k \in \mathcal{N} \cup\{N\}$.

2. The marginal rate $v_{k}^{\mathcal{N}_{k-1}}$ is nonincreasing in $k \in \mathcal{N}$.

The importance of PCL-indexability is due to the following main methodological result (see [14]). 
Proposition 4. If a problem is PCL-indexable with respect to the family of active sets $\mathcal{F}$ defined in (4), then it is indexable (i.e., the Whittle index exists) with index values $v_{n}:=v_{n}^{\mathcal{N}_{n-1}}$ for all $n \in \mathcal{N}$. Moreover, $\mathcal{F}$ contains an optimal policy for any value of parameter $v$.

The following proposition establishes the two assumptions given above to be sufficient for PCL-indexability. They are, however, not necessary, and thus the threshold policies defined in (4) may remain optimal even if our assumptions are violated. For all $n \in \mathcal{N}$, denote by $q_{n}:=\prod_{m=0}^{n-1} \beta p_{m}$. The proof consists of several auxiliary results for work and reward measures, and is deferred to the Appendices A-D.

Proposition 5. Under deteriorating QoS and concave adjusted reward, problem (2) is PCL-indexable with respect to the family $\mathcal{F}$ defined in (4). Moreover,

$$
\frac{R_{k}-\frac{p_{k}}{p_{k-1}} R_{k-1}}{W_{k}-\frac{p_{k}}{p_{k-1}} W_{k-1}} \leq v_{k}^{\mathcal{N}_{k-1}}=\frac{R_{k}+\sum_{m=0}^{k-1} q_{m+1}\left(R_{k}-\frac{p_{k}}{p_{m}} R_{m}\right)}{W_{k}+\sum_{m=0}^{k-1} q_{m+1}\left(W_{k}-\frac{p_{k}}{p_{m}} W_{m}\right)} \leq \frac{R_{k}}{W_{k}}
$$

where the lower bound holds as long as $W_{k}-\frac{p_{k}}{p_{k-1}} W_{k-1} \neq 0$.

Now we are ready to give a closed-form characterization of the transmission index. Let us define the transmission index as

$$
v_{n}:=\frac{R_{n}+\sum_{m=0}^{n-1} q_{m+1}\left(R_{n}-\frac{p_{n}}{p_{m}} R_{m}\right)}{W_{n}+\sum_{m=0}^{n-1} q_{m+1}\left(W_{n}-\frac{p_{n}}{p_{m}} W_{m}\right)} .
$$

The following theorem is the main result of this paper.

Theorem 1. Under deteriorating QoS and concave adjusted reward, problem (2) is

1. [Indexability] indexable with the index values given in (6);

2. [Optimality of Threshold Policies] optimally solved by a policy from the family $\mathcal{F}$ defined in (4).

Proof. By Propositions 5 and 4.

\subsection{Properties of transmission index}

Notice that for moderate values of the abandonment rate $\beta$, the expression of transmission index of state $n$ depends mostly on $W_{n}$ and $W_{m}$ 's for small values of $m$. The remaining parameters are less relevant since they appear multiplied by $\beta^{m+1}$ (via $q_{m+1}$ ). That stresses the importance of the initial values of the input stream, which was also identified by introducing slow start in the most TCP variants. The transmission index can be bounded by quantities independent of $\beta$; thus, these bounds are valid for flows with any abandonment rate.

Proposition 6. Under deteriorating QoS and concave adjusted reward, for any $\beta$,

$$
\frac{R_{n}-\frac{p_{n}}{p_{n-1}} R_{n-1}}{W_{n}-\frac{p_{n}}{p_{n-1}} W_{n-1}} \leq v_{n} \leq \frac{R_{n}}{W_{n}},
$$

where the lower bound holds as long as $W_{n}-\frac{p_{n}}{p_{n-1}} W_{n-1} \neq 0$.

Proof. By Propositions 5 and 4, and (6).

Note that if $W_{n}-\frac{p_{n}}{p_{n-1}} W_{n-1}=0$, then we can obtain a lower bound by replacing the terms of state $n-1$ by the terms of the largest state $m \leq n-2$ for which $W_{n}-\frac{p_{n}}{p_{m}} W_{m} \neq 0$. We further show that the transmission index is higher for flows with higher abandonment rate.

Proposition 7. The transmission index $v_{n}$ is nonincreasing in $\beta$. Further, $v_{n} \rightarrow \frac{R_{n}}{W_{n}}$ as $\beta \rightarrow 0$. 
Proof. Notice first in the transmission index expression (6) that $q_{m+1}$ depends multiplicatively on $\beta$. Further, as long as the two denominators below are nonzero, we have

$$
\frac{R_{n}-\frac{p_{n}}{p_{m}} R_{m}}{W_{n}-\frac{p_{n}}{p_{m}} W_{m}} \geq \frac{R_{n}-\frac{p_{n}}{p_{m+1}} R_{m+1}}{W_{n}-\frac{p_{n}}{p_{m+1}} W_{m+1}} \quad \text { for all } 0 \leq m \leq n-2
$$

by Lemma 9(iii). Therefore, Lemma 11 implies that the expression for the transmission index in Theorem 1 nondecreases when $\beta$ is decreased. The convergence as $\beta \rightarrow 0$ is obtained directly since $q_{m+1}$ depends multiplicatively on $\beta$.

\section{Optimal solution for single-bottleneck network}

Our model with $p_{n}=1$ for all $n \in \mathcal{N}$ recovers the traditional control problem in a single-bottleneck network, i.e., when there are no more routers downstream. The objective is to maximize a particular type of fairness, such as the generalized $\alpha$-fairness [4]. For any $\alpha \geq 0$, this is given by the function

$$
R(W):= \begin{cases}\frac{(1+W)^{1-\alpha}-1}{1-\alpha}, & \text { if } \alpha \neq 1, \\ \ln (1+W), & \text { if } \alpha=1 .\end{cases}
$$

Notice that such a function is non-negative, increasing, and concave on the domain of non-negative real numbers and $R(0)=0$. At $\alpha=1$ the general expression is substituted by its limit, which makes the function continuous in $\alpha$.

Suppose that the bottleneck router employs buffer tail dropping policy, and so the warning action is approximately performed by blocking the flow, i.e., $W_{n}^{0}=0$ for all $n \in \mathcal{N}$. Suppose further that the flow continuously increases the input stream whenever not warned, $0<W_{0}^{\text {sent }}<W_{1}^{\text {sent }}<\cdots<W_{N-1}^{\text {sent }}$. Since $W_{n}^{1}=W_{n}^{\text {sent }}$ is the number of packets sent if the flow is in state $n$ (i.e., the actual input rate at the router), the congestion-free reward simplifies to $R_{n}^{1}=R_{n}^{1+}=R\left(W_{n}^{1}\right)$. On the other hand, $R_{n}^{0}=R_{n}^{1-}=R\left(W_{n}^{0}\right)=0$.

Using (3), we have $W_{n}=W_{n}^{1}$ and $R_{n}=R_{n}^{1}$. In addition, we denote by $W_{-1}:=0$. We can then readily apply the results of the previous section to obtain the following characterization of the transmission index:

$$
v_{n}= \begin{cases}\frac{\sum_{m=0}^{n} \beta^{m}\left[\left(1+W_{n}\right)^{1-\alpha}-\left(1+W_{m-1}\right)^{1-\alpha}\right]}{(1-\alpha)\left(\sum_{m=0}^{n} \beta^{m}\left[W_{n}-W_{m-1}\right]\right)}, & \text { if } \alpha \neq 1, \\ \frac{\sum_{m=0}^{n} \beta^{m}\left[\ln \left(1+W_{n}\right)-\ln \left(1+W_{m-1}\right)\right]}{\sum_{m=0}^{n} \beta^{m}\left[W_{n}-W_{m-1}\right]}, & \text { if } \alpha=1 .\end{cases}
$$

\section{Numerical example}

In this section we illustrate the optimal index policy numerically. Consider a double-bottleneck network as in Fig. 1. As above, suppose that Router 1 employs buffer tail dropping policy, and so the warning action is approximately performed by blocking the flow, i.e., $W_{n}^{0}=0$ for all $n \in \mathcal{N}$. Suppose that Router2 has implemented a random packet dropping/marking policy (e.g., RED) with probability $p$ of dropping/marking a packet. That is, the flow data in state $n$ transmitted without warning at Router 1 is transmitted without warning also at Router 2 with probability $p_{n}=(1-p)^{W_{n}^{1}}$. We assume $\beta=1$.

Consider a flow with $N=6$ states and with sending rate $\boldsymbol{W}^{\text {sent }}:=(1,2,4,8,9,10)$. Thus, the first three states follow an exponential growth (known as TCP's slow-start phase), while the remaining three states follow a linear growth (known as congestion avoidance phase). Suppose that the transmission cost is $v=0.18$ at Router 1 , where the policy is to be implemented in order to achieve proportional fairness $(\alpha=1)$.

If there is no congestion at Router2, i.e., $p=0$, then the index values are

$$
(0.6931,0.5014,0.3374,0.2155,0.1972,0.1802) \text {. }
$$

The flow data are transmitted without warning in all the states, because the index values are all larger than the transmission cost.

If there is certain congestion at Router 2 represented by $p=0.02$, then the index values become

$(0.6793,0.4790,0.3078,0.1804,0.1617,0.1450)$.

All the index values are now lower than in the no-congestion case. In particular, the flow data are transmitted without warning in the first four states, but it is warned in the last two states. 
If the flow were more aggressive and growing exponentially in all the states with $\boldsymbol{W}^{\text {sent }}:=(1,2,4,8,16$, 32), while keeping $p=0$, then the index values only change in the last two states and become 0.1318 and 0.0779 , respectively. The flow would be warned in the last two states, since those index values are below the transmission cost.

Note that it can be numerically checked that the above parameters satisfy the two assumptions required in our analysis.

\section{Conclusion and further research directions}

In this paper we have focused on particular type of TCP flows with any-increase/restarting dynamics, which roughly resembles the behavior of currently common TCP variants such as CUBIC and COMPOUND. Other flow types are likely to be tractable in our framework with moderate modifications. Our analytical approach is very flexible. By means of the transmission index we have obtained optimal solution that can be interpreted as the network price of the flow and proposes itself to be implemented in congestion notification mechanisms to improve their resource allocation decisions.

From the modeling perspective, several challenges remain open. For instance, our model assumes that the congestion information is instantaneously received by network nodes. A more realistic model would take into account information delays and censoring due to unobserved dropped packets. Existence of finite buffers and non-cooperative flows further raises the necessity of consideration of three actions at the router: transmitting, warning, and dropping. However, index policies have only been obtained in the literature for optimization problems with two actions, such as transmitting and warning as we have considered here. A possible approach for multi-action problems was outlined in [28].

From the practical point of view, however, we believe that the outcome of our model is roughly preserved also in more complicated mechanisms. The reason being that the transmission index depends on the actual input stream and the slow start values much more strongly than on other aspects of the dynamics of the mechanism. Besides the congestion notification mechanisms on which we have focused in this paper, the transmission index could be also implemented in scheduling algorithms. The use of transmission index values as weights in weighted fair queueing would imply that each flow data are transmitted accordingly to their actual network price.

We could also take advantage of our approach turning it upside-down. Instead of improving congestion notification mechanisms for a given protocol, we could set out to develop a protocol that is economically sound given a congestion notification mechanism. The task would then be to formulate a protocol for which the transmission index would have desirable properties. Some numerical results in this direction were presented by Handley et al. [29].

\section{Appendix A. Notation}

We stick to the following norms throughout the paper. Sets are typeset in calligraphic font (e.g., $\mathcal{N}$ ), their cardinality is denoted by the corresponding uppercase letter $(N)$, and their generic element is written in lowercase $(n)$. Vectors are in boldface in row/column form as convenient, and matrices are in uppercase boldface. For problem parameters and variables, superscript is reserved to an action or policy, and subscript to a state.

Sets:

$$
\begin{aligned}
& \mathcal{N}:=\{0,1, \ldots, N-1\} \text {-state space of the MDP model } \\
& \mathcal{N}_{k}:=\{n \in \mathcal{N}: n \leq k\} \\
& s \subseteq \mathcal{N} \text {-control policy (active set, i.e., set of states in which flow data are transmitted) } \\
& \mathcal{F} \subseteq 2^{\mathcal{N}} \text {-family of control policies (active sets). }
\end{aligned}
$$

Problem parameters:
$R_{n}$-reward earned if flow data in state $n$ are transmitted
$W_{n}>0$-required bandwidth utilization (or work) if flow data in state $n$ are transmitted
$\nu$-transmission cost per bandwidth unit
$0<\beta<1-$ discount factor.

Elements in work-reward analysis:

$$
\begin{aligned}
& \mathbb{R}_{n}^{s} \text {-expected total } \beta \text {-discounted reward under policy } \& \text { if starting from state } n \\
& \mathbb{W}_{n}^{\&} \text {-expected total } \beta \text {-discounted work under policy } \& \text { if starting from state } n \\
& r_{n}^{s} \text {-marginal reward under policy } \& \text { if starting from state } n \\
& w_{n}^{8} \text {-marginal work under policy } \& \text { if starting from state } n \\
& v_{n}^{8}:=r_{n}^{s} / w_{n}^{s} \text {-marginal rate under policy } \& \text { if starting from state } n \\
& v_{n} \text {-transmission index of state } n \text {. }
\end{aligned}
$$




\section{Appendix B. Normalization of the optimization problem}

Next we develop the matrices needed for normalization of the model. We have

$$
\boldsymbol{P}^{0}=\begin{gathered}
0 \\
1 \\
2 \\
\vdots \\
N-1
\end{gathered}\left(\begin{array}{ccccc}
1 & 0 & 0 & 0 & 0 \\
1 & 0 & 0 & 0 & 0 \\
1 & 0 & 0 & 0 & 0 \\
1 & 0 & 0 & \ddots & 0 \\
1 & 0 & 0 & 0 & 0
\end{array}\right),
$$

and therefore

$$
\boldsymbol{I}-\beta \boldsymbol{P}^{0}=\underset{N-1}{0} \begin{gathered}
1 \\
2 \\
\vdots \\
-\beta
\end{gathered}\left(\begin{array}{ccccc}
1-\beta & 0 & 0 & 0 & 0 \\
-\beta & 1 & 0 & 0 & 0 \\
-\beta & 0 & 1 & 0 & 0 \\
-\beta & 0 & 0 & \ddots & 0 \\
-\beta & 0 & 0 & 0 & 1
\end{array}\right) .
$$

It is easy to see that $\boldsymbol{I}-\beta \boldsymbol{P}^{0}$ is invertible if and only if $\beta \neq 1$, and the inverse is

$$
\left(\boldsymbol{I}-\beta \boldsymbol{P}^{0}\right)^{-1}=\begin{gathered}
0 \\
1 \\
2 \\
\vdots \\
N-1
\end{gathered}\left(\begin{array}{ccccc}
\frac{1}{1-\beta} & 0 & 0 & 0 & 0 \\
\frac{\beta}{1-\beta} & 1 & 0 & 0 & 0 \\
\frac{\beta}{1-\beta} & 0 & 1 & 0 & 0 \\
\frac{\beta}{1-\beta} & 0 & 0 & \ddots & 0 \\
\frac{\beta}{1-\beta} & 0 & 0 & 0 & 1
\end{array}\right) .
$$

Further,

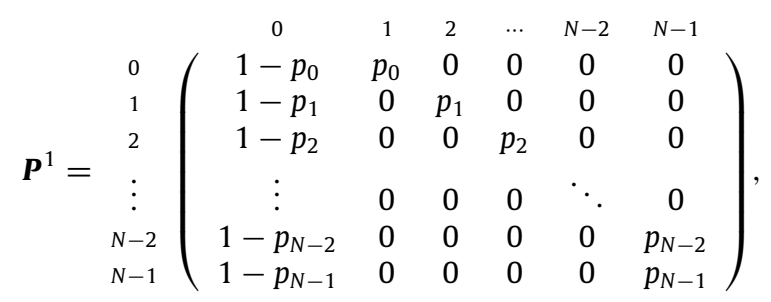

and therefore

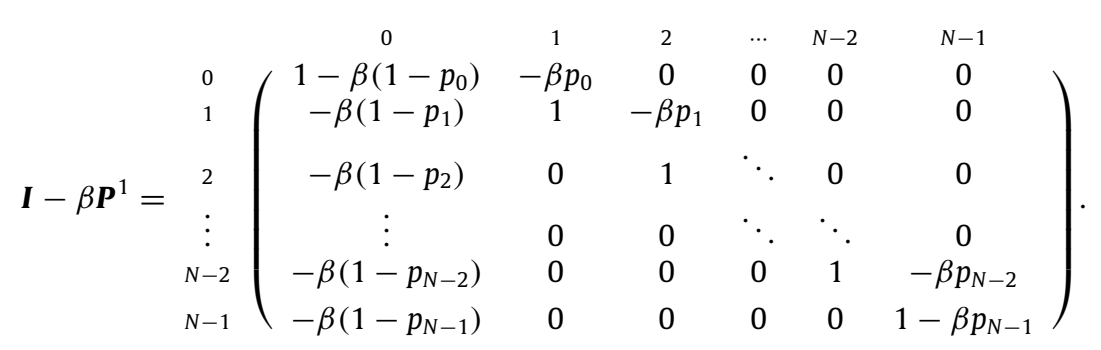

Finally,

$$
\left(\boldsymbol{I}-\beta \boldsymbol{P}^{1}\right)\left(\boldsymbol{I}-\beta \boldsymbol{P}^{0}\right)^{-1}=\begin{gathered}
0 \\
1 \\
2 \\
\vdots \\
N-2 \\
N-1
\end{gathered}\left(\begin{array}{cccccc}
1+\beta p_{0} & -\beta p_{0} & 0 & 0 & 0 & 0 \\
\beta p_{1} & 1 & -\beta p_{1} & 0 & 0 & 0 \\
\beta p_{2} & 0 & 1 & \ddots & 0 & 0 \\
\vdots & 0 & 0 & \ddots & \ddots & 0 \\
\beta p_{N-2} & 0 & 0 & 0 & 1 & -\beta p_{N-2} \\
\beta p_{N-1} & 0 & 0 & 0 & 0 & 1-\beta p_{N-1}
\end{array}\right) .
$$


Thus, for the normalized formulation we set, for all $n \in \mathcal{N}$,

$$
R_{n}:=\left(R_{n}^{1}-R_{n}^{0}\right)+\beta p_{n}\left(R_{n+1}^{0}-R_{0}^{0}\right), \quad W_{n}:=\left(W_{n}^{1}-W_{n}^{0}\right)+\beta p_{n}\left(W_{n+1}^{0}-W_{0}^{0}\right)
$$

where we have defined $R_{N}^{0}:=R_{N-1}^{0}$ and $W_{N}^{0}:=W_{N-1}^{0}$.

\section{Appendix C. Work-reward analysis and proof of Proposition 5}

To achieve the goal, we first characterize the marginal reward and marginal work. For all $n \in \mathcal{N}$, let $Q_{n}:=\sum_{m=0}^{n}$ $q_{m}, Q_{n}^{(R)}:=\sum_{m=0}^{n} q_{m} R_{m}$, and $Q_{n}^{(W)}:=\sum_{m=0}^{n} q_{m} W_{m}$.

Lemma 2. For $k, n \in \mathcal{N}$ with $n \geq k-1$, the marginal reward and work, respectively, are

$$
r_{n}^{\mathcal{N}_{k-1}}=R_{n}-\beta p_{n} \frac{Q_{k-1}^{(R)}}{Q_{k}}, \quad w_{n}^{\mathcal{N}_{k-1}}=W_{n}-\beta p_{n} \frac{Q_{k-1}^{(W)}}{Q_{k}} .
$$

Proof. For $k=0$ consider $\mathcal{N}_{-1}=\emptyset$. Then for any state $n \in \mathcal{N}$ we have $\mathbb{R}_{n}^{\mathcal{N}_{-1}}=\mathbb{W}_{n}^{\mathcal{N}_{-1}}=0$, and hence $r_{n}^{\mathcal{N}_{-1}}=R_{n}$ and $w_{n}^{\mathcal{N}_{-}}=W_{n}$ by Proposition 2 .

Now for $k \in \mathcal{N} \backslash\{0\}$ consider $\mathcal{N}_{k-1}$. Using Proposition 1 , for $n \leq k-1$ we have

$$
\mathbb{R}_{n}^{\mathcal{N}_{k-1}}=R_{n}+\beta\left(p_{n} \mathbb{R}_{n+1}^{\mathcal{N}_{k-1}}+\left(1-p_{n}\right) \mathbb{R}_{0}^{\mathcal{N}_{k-1}}\right), \quad \mathbb{W}_{n}^{\mathcal{N}_{k-1}}=W_{n}+\beta\left(p_{n} \mathbb{W}_{n+1}^{\mathcal{N}_{k-1}}+\left(1-p_{n}\right) \mathbb{W}_{0}^{\mathcal{N}_{k-1}}\right),
$$

and for $n \geq k$ we have $\mathbb{R}_{n}^{\mathcal{N}_{k-1}}=\beta \mathbb{R}_{0}^{\mathcal{N}_{k-1}}$ and $\mathbb{W}_{n}^{\mathcal{N}_{k-1}}=\beta \mathbb{W}_{0}^{\mathcal{N}_{k-1}}$.

The solution of the two above linear-equation systems gives

$$
(1-\beta) \mathbb{R}_{0}^{\mathcal{N}_{k-1}}=\frac{Q_{k-1}^{(R)}}{Q_{k}}, \quad(1-\beta) \mathbb{W}_{0}^{\mathcal{N}_{k-1}}=\frac{Q_{k-1}^{(W)}}{Q_{k}} .
$$

Therefore, we have $\mathbb{R}_{n}^{\mathcal{N}_{k-1}}-\mathbb{R}_{0}^{\mathcal{N}_{k-1}}=-(1-\beta) \mathbb{R}_{0}^{\mathcal{N}_{k-1}}$ and $\mathbb{W}_{n}^{\mathcal{N}_{k-1}}-\mathbb{W}_{0}^{\mathcal{N}_{k-1}}=-(1-\beta) \mathbb{W}_{0}^{\mathcal{N}_{k-1}}$ for $n \geq k$. Then Proposition 2 yields (C.1) for $k, n \in \mathcal{N}$ with $n \geq k-1$.

In the following lemma we establish positivity of the marginal works as required in condition (i) of $\operatorname{PCL}(\mathcal{F})$-indexability.

Lemma 3. Under deteriorating QoS, the marginal work $w_{n}^{\mathcal{N}_{k-1}}$ is positive for all $n \in \mathcal{N}$ and $k \in \mathcal{N} \cup\{N\}$, i.e., condition (i) of $\operatorname{PCL}(\mathcal{F})$-indexability holds.

Proof. If $k \neq N$, then the marginal work $w_{n}^{\mathcal{N}_{k-1}}$ is positive for $n \geq k-1$, because from (C.1) it can be rewritten as

$$
w_{n}^{\mathcal{N}_{k-1}}=\frac{1}{Q_{k}}\left(W_{n}+\sum_{m=0}^{k-1} q_{m+1}\left(W_{n}-\frac{p_{n}}{p_{m}} W_{m}\right)\right),
$$

which is positive due to deteriorating QoS.

For $n \leq k-1 \leq N-1$ we proceed as follows. Proposition 1 after rearranging gives, for $n \geq 1$,

$$
\mathbb{W}_{n}^{\mathcal{N}_{k-1}}=\left(\mathbb{W}_{n-1}^{\mathcal{N}_{k-1}}-\beta\left(1-p_{n-1}\right) \mathbb{W}_{0}^{\mathcal{N}_{k-1}}-W_{n-1}\right) / \beta p_{n-1},
$$

whose recursive implementation yields

$$
\mathbb{W}_{n}^{\mathcal{N}_{k-1}}=\frac{Q_{n}-\beta Q_{n-1}}{q_{n}} \mathbb{W}_{0}^{\mathcal{N}_{k-1}}-\frac{Q_{n-1}^{(W)}}{q_{n}} .
$$

Due to Proposition 3, we want to prove $\mathbb{W}_{n}^{\mathcal{N}_{k-1}}>\beta \mathbb{W}_{0}^{\mathcal{N}_{k-1}}$, i.e.,

$$
(1-\beta) \mathbb{W}_{0}^{\mathcal{N}_{k-1}}>\frac{Q_{n-1}^{(W)}}{Q_{n}} .
$$

For $k=N$, (C.5) evaluated for $n=N-1$ together with Proposition 1 gives

$$
(1-\beta) \mathbb{W}_{0}^{\mathcal{N}_{N-1}}=\frac{Q_{N-2}^{(W)}+W_{N-1} \frac{q_{N-1}}{1-\beta p_{N-1}}}{Q_{N-1}+\beta p_{N-1} \frac{q_{N-1}}{1-\beta p_{N-1}}} .
$$

For $k \neq N$, we use (C.2). In both cases, deteriorating QoS and Lemma 7 imply (C.6). 
Next we characterize and bound the marginal rates $v_{k}^{\mathcal{N}_{k-1}}$, which will be crucial for the characterization of the transmission index in the next subsection.

Lemma 4. Under concave adjusted reward and deteriorating $Q o S$, for $k \in \mathcal{N}$, we have

$$
\frac{R_{k}-\frac{p_{k}}{p_{k-1}} R_{k-1}}{W_{k}-\frac{p_{k}}{p_{k-1}} W_{k-1}} \leq v_{k}^{\mathcal{N}_{k-1}}=\frac{R_{k}+\sum_{m=0}^{k-1} q_{m+1}\left(R_{k}-\frac{p_{k}}{p_{m}} R_{m}\right)}{W_{k}+\sum_{m=0}^{k-1} q_{m+1}\left(W_{k}-\frac{p_{k}}{p_{m}} W_{m}\right)} \leq \frac{R_{k}}{W_{k}},
$$

where the lower bound holds as long as $W_{k}-\frac{p_{k}}{p_{k-1}} W_{k-1} \neq 0$.

Proof. The expression for $v_{k}^{\mathcal{N}_{k-1}}$ is a simple reformulation of (C.1), as in (C.3). Having $R_{m} / W_{m} \geq R_{k} / W_{k}$ for all $m<k$ from Lemma 10(ii), we can rewrite it as

$$
\frac{R_{k}-\frac{p_{k}}{p_{m}} R_{m}}{W_{k}-\frac{p_{k}}{p_{m}} W_{m}} \leq \frac{R_{k}}{W_{k}}
$$

as long as $W_{k}-\frac{p_{k}}{p_{m}} W_{m} \neq 0$ (otherwise, the $m$-th term in the sum is zero both in the numerator and denominator). Then, Lemma 8 yields the upper bound for $v_{k}^{\mathcal{N}_{k-1}}$.

We prove the lower bound next. Lemma 8 together with the upper bound implies

$$
\min \left\{\frac{R_{k}-\frac{p_{k}}{p_{m}} R_{m}}{W_{k}-\frac{p_{k}}{p_{m}} W_{m}} \text { for all } 0 \leq m \leq k-1 \text { such that } W_{k}-\frac{p_{k}}{p_{m}} W_{m} \neq 0\right\} \leq v_{k}^{\mathcal{N}_{k-1}} .
$$

Further, if $W_{k}-\frac{p_{k}}{p_{k-1}} W_{k-1} \neq 0$, then we have

$$
\frac{R_{k}-\frac{p_{k}}{p_{m}} R_{m}}{W_{k}-\frac{p_{k}}{p_{m}} W_{m}} \geq \frac{R_{k}-\frac{p_{k}}{p_{k-1}} R_{k-1}}{W_{k}-\frac{p_{k}}{p_{k-1}} W_{k-1}} \text { for all } 0 \leq m \leq k-1
$$

as long as $W_{k}-\frac{p_{k}}{p_{m}} W_{m} \neq 0$ by Lemma 9(iii), which gives the lower bound.

Further, we will need the following monotonicity result for marginal rates.

Lemma 5. Under concave adjusted reward and deteriorating $Q o S, v_{k}^{\mathcal{N}_{k-1}} \geq v_{n}^{\mathcal{N}_{k-1}}$ for all $k, n \in \mathcal{N}$ with $n \geq k+1$.

Proof. Denote by

$$
a_{k}:=\frac{\beta Q_{k-1}^{(R)}}{Q_{k}}, \quad b_{k}:=\frac{\beta Q_{k-1}^{(W)}}{Q_{k}} .
$$

Using the identities in (C.1), we are to show that

$$
v_{k}^{\mathcal{N}_{k-1}}=\frac{\frac{R_{k}}{p_{k}}-a_{k}}{\frac{W_{k}}{p_{k}}-b_{k}} \geq \frac{\left(\frac{R_{k}}{p_{k}}-a_{k}\right)+\left(\frac{R_{n}}{p_{n}}-\frac{R_{k}}{p_{k}}\right)}{\left(\frac{W_{k}}{p_{k}}-b_{k}\right)+\left(\frac{W_{n}}{p_{n}}-\frac{W_{k}}{p_{k}}\right)}=\frac{\frac{R_{n}}{p_{n}}-a_{k}}{\frac{W_{n}}{p_{n}}-b_{k}}=v_{n}^{\mathcal{N}_{k-1}} \quad \text { for all } N-1 \geq n \geq k+1 .
$$

If $\frac{W_{n}}{p_{n}}=\frac{W_{k}}{p_{k}}$, then it holds as equality. Otherwise, having

$$
\frac{\frac{R_{k}}{p_{k}}-a_{k}}{\frac{W_{k}}{p_{k}}-b_{k}} \geq \frac{\frac{R_{n}}{p_{n}}-\frac{R_{k}}{p_{k}}}{\frac{W_{n}}{p_{n}}-\frac{W_{k}}{p_{k}}} \quad \text { for all } N-1 \geq n \geq k+1
$$

is equivalent to

$$
\frac{\frac{R_{k}}{p_{k}}-a_{k}}{\frac{W_{k}}{p_{k}}-b_{k}} \geq \frac{\frac{R_{k+1}}{p_{k+1}}-\frac{R_{k}}{p_{k}}}{\frac{W_{k+1}}{p_{k+1}}-\frac{W_{k}}{p_{k}}}
$$

since the right-hand side is nonincreasing in $n$, due to concave adjusted reward with Lemma 9 (ii). The last inequality holds due to the lower bound in Lemma 4 and, again, concave adjusted reward with Lemma 9(iv). Therefore, by Lemma 7(ii), (C.12) holds. 
Next we establish condition (ii) of $\operatorname{PCL}(\mathcal{F})$-indexability.

Lemma 6. Under concave adjusted reward and deteriorating QoS, marginal rates $v_{n}^{\mathcal{N}_{n-1}}$ are nonincreasing in $n \in \mathcal{N}$, i.e., condition (ii) of $\mathrm{PCL}(\mathcal{F})$-indexability holds.

Proof. Niño-Mora [14, Proposition 6.4(c)] showed that under positive marginal works, $v_{n}^{\mathcal{N}_{n-1}} \geq v_{n+1}^{\mathcal{N}_{n}}$ is equivalent to $v_{n}^{\mathcal{N}_{n-1}} \geq v_{n+1}^{\mathcal{N}_{n-1}}$, which is satisfied due to Lemma 5 .

To summarize, in this section we have proved Proposition 5.

\section{Appendix D. Auxiliary results}

Some of the following lemmas are well-known, see for instance [31].

Lemma 7. Let $b_{0}, b_{1}, \alpha_{1}>0$. Then the following statements are equivalent:

1. $\frac{a_{0}}{b_{0}} \geq \frac{a_{1}}{b_{1}}$;

2. $\frac{a_{0}}{b_{0}} \geq \frac{a_{0}+\alpha_{1} a_{1}}{b_{0}+\alpha_{1} b_{1}}$;

3. $\frac{a_{0}+\alpha_{1} a_{1}}{b_{0}+\alpha_{1} b_{1}} \geq \frac{a_{1}}{b_{1}}$.

Further, the above equivalence holds also if all inequalities are strict.

Proof. A simple algebraic exercise.

Lemma 8. Let for a positive integer $K$ be $\frac{a_{0}}{b_{0}} \geq \frac{a_{1}}{b_{1}} \geq \cdots \geq \frac{a_{K}}{b_{K}}$ such that $b_{0}, b_{1}, \ldots, b_{K}>0$ and $\alpha_{1}, \ldots, \alpha_{K} \geq 0$. Then

$$
\frac{a_{0}}{b_{0}} \geq \frac{a_{0}+\alpha_{1} a_{1}+\cdots+\alpha_{K} a_{K}}{b_{0}+\alpha_{1} b_{1}+\cdots+\alpha_{K} b_{K}} \geq \frac{a_{K}}{b_{K}} .
$$

Proof. For $K=1$ see Lemma 7. To proceed by induction, suppose that the claim holds for all $k=1,2, \ldots, K-1$. If $\alpha_{1}=0$, then we are in the case $k=K-1$, so suppose $\alpha_{1}>0$. Then, by the induction assumption for $k=K-1$, we have

$$
\frac{a_{1}}{b_{1}} \geq \frac{a_{1}+\frac{\alpha_{2}}{\alpha_{1}} a_{2}+\cdots+\frac{\alpha_{K}}{\alpha_{1}} a_{K}}{b_{1}+\frac{\alpha_{2}}{\alpha_{1}} b_{2}+\cdots+\frac{\alpha_{K}}{\alpha_{1}} b_{K}} .
$$

Since $\frac{a_{0}}{b_{0}} \geq \frac{a_{1}}{b_{1}}$, we have $\frac{a_{0}}{b_{0}}$ larger than or equal to the right-hand side of (D.2). Applying Lemma 7 gives

$$
\frac{a_{0}}{b_{0}} \geq \frac{a_{0}+\alpha_{1} a_{1}+\cdots+\alpha_{K} a_{K}}{b_{0}+\alpha_{1} b_{1}+\cdots+\alpha_{K} b_{K}} \geq \frac{a_{1}+\frac{\alpha_{2}}{\alpha_{1}} a_{2}+\cdots+\frac{\alpha_{K}}{\alpha_{1}} a_{K}}{b_{1}+\frac{\alpha_{2}}{\alpha_{1}} b_{2}+\cdots+\frac{\alpha_{K}}{\alpha_{1}} b_{K}},
$$

where the right-hand side expression is, again by the induction assumption for $k=K-1$, larger than or equal to $\frac{a_{K}}{b_{K}}$. This completes the proof for $k=K$.

Lemma 9 (Equivalent Definitions of Concavity). Consider a real-valued function $a(\cdot)$, a positive integer $K$, and a set $B$ := $\left\{0, b_{0}, b_{1}, \ldots, b_{K}\right\}$ such that $0<b_{0}<b_{1}<\ldots<b_{K}$. Denote by $a_{k}:=a\left(b_{k}\right)$ for any $k=0,1, \ldots, K$. Then the following statements are equivalent:

1. the function $a(\cdot)$ is concave on $\mathcal{B}$;

2. $\frac{a_{k_{1}}-a_{k_{0}}}{b_{k_{1}}-b_{k_{0}}} \geq \frac{a_{k_{2}}-a_{k_{0}}}{b_{k_{2}}-b_{k_{0}}}$ for any $b_{k_{0}}, b_{k_{1}}, b_{k_{2}} \in \mathcal{B}$ with $b_{k_{0}}<b_{k_{1}}<b_{k_{2}}$;

3. $\frac{a_{k_{2}}-a_{k_{0}}}{b_{k_{2}}-b_{k_{0}}} \geq \frac{a_{k_{2}}-a_{k_{1}}}{b_{k_{2}}-b_{k_{1}}}$ for any $b_{k_{0}}, b_{k_{1}}, b_{k_{2}} \in \mathcal{B}$ with $b_{k_{0}}<b_{k_{1}}<b_{k_{2}}$;

4. $\frac{a_{k_{1}}-a_{k_{0}}}{b_{k_{1}}-b_{k_{0}}} \geq \frac{a_{k_{2}}-a_{k_{1}}}{b_{k_{2}}-b_{k_{1}}}$ for any $b_{k_{0}}, b_{k_{1}}, b_{k_{2}} \in \mathcal{B}$ with $b_{k_{0}}<b_{k_{1}}<b_{k_{2}}$.

Lemma 10. Let $a$ be a concave real-valued function with $a(0) \geq 0$. Consider a positive integer $K$ and $a$ set $\mathscr{B}:=\left\{0, b_{0}\right.$, $\left.b_{1}, \ldots, b_{K}\right\}$ such that $0<b_{0}<b_{1}<\cdots<b_{K}$. Denote by $a_{k}:=a\left(b_{k}\right)$ for any $k=0,1, \ldots, K$. Then

1. $\frac{a_{k_{2}}}{b_{k_{2}}} \geq \frac{a_{k_{2}}-a_{k_{1}}}{b_{k_{2}}-b_{k_{1}}}$ for any $b_{k_{1}}, b_{k_{2}} \in \mathcal{B}$ with $0<b_{k_{1}}<b_{k_{2}}$;

2. $\frac{a_{0}}{b_{0}} \geq \frac{a_{1}}{b_{1}} \geq \cdots \geq \frac{a_{K}}{b_{K}}$;

3. $\frac{a_{k_{1}}}{b_{k_{1}}} \geq \frac{a_{k_{2}}-a_{k_{1}}}{b_{k_{2}}-b_{k_{1}}}$ for any $b_{k_{1}}, b_{k_{2}} \in \mathscr{B}$ with $0<b_{k_{1}}<b_{k_{2}}$. 
Proof. 1 . We start by setting $b_{k_{0}}=0$ in Lemma 9(iii). Hence, for any $k_{1} \in\{0,1, \ldots, K-1\}$,

$$
\frac{a_{k_{2}}}{b_{k_{2}}} \geq \frac{a_{k_{2}}-a(0)}{b_{k_{2}}} \geq \frac{a_{k_{2}}-a_{k_{1}}}{b_{k_{2}}-b_{k_{1}}}
$$

where the first inequality is due to $a(0) \geq 0$.

2. By rearranging the terms in (i) we obtain $a_{k_{1}} / b_{k_{1}} \geq a_{k_{2}} / b_{k_{2}}$ for any $k_{1} \in\{0,1, \ldots, K-1\}$ and $k_{2}>k_{1}$.

3. Since (i) holds and by (ii), $a_{k_{1}} / b_{k_{1}} \geq a_{k_{2}} / b_{k_{2}}$, the result is immediate.

Lemma 11. Let for a positive integer $K$ be $\frac{a_{0}}{b_{0}} \geq \frac{a_{1}}{b_{1}} \geq \cdots \geq \frac{a_{K}}{b_{K}}$ such that $b_{0}, b_{1}, \ldots, b_{K}>0$. Let $\alpha_{1}, \ldots, \alpha_{K} \geq 0$ and $1 \geq \gamma_{1}>\gamma_{2}>\cdots>\gamma_{K} \geq 0$. Then

$$
\frac{a_{0}+\alpha_{1} a_{1}+\cdots+\alpha_{K} a_{K}}{b_{0}+\alpha_{1} b_{1}+\cdots+\alpha_{K} b_{K}} \leq \frac{a_{0}+\gamma_{1} \alpha_{1} a_{1}+\cdots+\gamma_{K} \alpha_{K} a_{K}}{b_{0}+\gamma_{1} \alpha_{1} b_{1}+\cdots+\gamma_{K} \alpha_{K} b_{K}} .
$$

Proof. Consider $k=1$ and $\gamma_{1}<1$ (for $\gamma_{1}=1$ it trivially holds). Hence, we can multiply $\frac{a_{0}}{b_{0}} \geq \frac{a_{1}}{b_{1}}$ by the positive expression $\alpha_{1}\left(1-\gamma_{1}\right) b_{0} b_{1}$, and add $a_{0} b_{0}+\gamma_{1} \alpha_{1}^{2} a_{1} b_{1}$, which after rearranging gives the desired result. To proceed by induction, suppose that the claim holds for all $k=1,2, \ldots, K-1$, and suppose $\alpha_{1}>0$ (otherwise it is true by the induction assumption). Denote

$$
d_{a}:=a_{1}+\frac{\gamma_{2}}{\gamma_{1}} \frac{\alpha_{2}}{\alpha_{1}} a_{2}+\cdots+\frac{\gamma_{K}}{\gamma_{1}} \frac{\alpha_{K}}{\alpha_{1}} a_{K}, \quad d_{b}:=b_{1}+\frac{\gamma_{2}}{\gamma_{1}} \frac{\alpha_{2}}{\alpha_{1}} b_{2}+\cdots+\frac{\gamma_{K}}{\gamma_{1}} \frac{\alpha_{K}}{\alpha_{1}} b_{K},
$$

and further denote by $c_{a}$ and $c_{b}$ the expressions obtained from $d_{a}$ and $d_{b}$ by omitting $\gamma$ 's. Note that we want to show

$$
\frac{a_{0}+\alpha_{1} c_{a}}{b_{0}+\alpha_{1} c_{b}} \leq \frac{a_{0}+\gamma_{1} \alpha_{1} d_{a}}{b_{0}+\gamma_{1} \alpha_{1} d_{b}}
$$

Using Lemma 8 and multiplying both numerator and denominator by $\alpha_{1}\left(1-\gamma_{1}\right)$ we obtain

$$
\frac{a_{1}}{b_{1}} \geq \frac{\alpha_{1}\left(1-\gamma_{1}\right) a_{1}+\alpha_{2}\left(1-\gamma_{2}\right) a_{2}+\cdots+\alpha_{K}\left(1-\gamma_{K}\right) a_{K}}{\alpha_{1}\left(1-\gamma_{1}\right) b_{1}+\alpha_{2}\left(1-\gamma_{2}\right) b_{2}+\cdots+\alpha_{K}\left(1-\gamma_{K}\right) b_{K}}
$$

and therefore we can write

$$
\frac{a_{0}}{b_{0}} \geq \frac{\alpha_{1} c_{a}-\gamma_{1} \alpha_{1} d_{a}}{\alpha_{1} c_{b}-\gamma_{1} \alpha_{1} d_{b}}
$$

which is the same as $a_{0}\left(\alpha_{1} c_{b}-\gamma_{1} \alpha_{1} d_{b}+b_{0}\right) \geq b_{0}\left(\alpha_{1} c_{a}-\gamma_{1} \alpha_{1} d_{a}+a_{0}\right)$.

On the other hand, the induction assumption implies $\frac{c_{a}}{c_{b}} \leq \frac{d_{a}}{d_{b}}$, hence $0 \geq \alpha_{1}^{2} \gamma_{1}\left(c_{a} d_{b}-d_{a} c_{b}\right)$. Adding up the last two inequalities and rearranging yields (D.5).

\section{References}

[1] S. Floyd, K. Fall, Promoting the use of end-to-end congestion control in the Internet, IEEE/ACM Transactions on Networking 7 (1999) $458-472$.

[2] D. Wischik, How to mark fairly, Manuscript, 1999.

[3] J. Mo, J. Walrand, Fair end-to-end window-based congestion control, IEEE/ACM Transactions on Networking 8 (2000) 556-567.

[4] E. Altman, K. Avrachenkov, A. Garnaev, Generalized $\alpha$-fair resource allocation in wireless networks, in: Proceedings of the 47th IEEE Conference on Decision and Control, Cancun, Mexico, pp. 2414-2419.

[5] P. Whittle, Restless bandits: activity allocation in a changing world, in: J. Gani (Ed.), A Celebration of Applied Probability, Journal of Applied Probability 25A (1988), 287-298.

[6] B. Braden, D. Clark, J. Crowcroft, B. Davie, S. Deering, D. Estrin, S. Floyd, V. Jacobson, G. Minshall, C. Partridge, L. Peterson, K. Ramakrishnan, S. Shenker, J. Wroclawski, L. Zhang, RFC 2309: recommendations on queue management and congestion avoidance in the Internet, 1998. Available as RFC 2309 Ftp://ftp.isi.edu/in-notes/rfc2309.txt.

[7] S. Floyd, V. Jacobson, Random early detection gateways for congestion avoidance, IEEE/ACM Transactions on Networking 1 (1993) $397-413$.

[8] W. Feng, K.G. Shin, D.D. Kandlur, D. Saha, The BLUE active queue management algorithms, IEEE/ACM Transactions on Networking 10 (2002) 513-528.

[9] M.J. Neely, E. Modiano, C.P. Li, Fairness and optimal stochastic control for heterogeneous networks, IEEE/ACM Transactions on Networking 16 (2008) 396-409.

[10] F. Paganini, Congestion control with adaptive multipath routing based on optimization, in: Proceedings of the Conference on Information Sciences and Systems, pp. 333-338.

[11] M. Molle, Z. Xu, Short-circuiting the congestion signaling path for AQM algorithms using reverse flow matching, Computer Communications 28 (2005) 2082-2093.

[12] L. Massoulié, J. Roberts, Arguments in favour of admission control for TCP flows, in: Teletraffic Engineering in a Competitive World, Proceedings of ITC 16, pp. 33-44.

[13] P. Naor, The regulation of queue size by levying tolls, Econometrica 37 (1969) 15-24.

[14] J. Niño-Mora, Dynamic allocation indices for restless projects and queueing admission control: a polyhedral approach, Mathematical Programming. Series A 93 (2002) 361-413.

[15] A.B. Piunovskiy, Bicriteria optimization of a queue with a controlled input stream, Queueing Systems 48 (2004) 159-184.

[16] A. Ferragut, F. Paganini, Achieving network stability and user fairness through admission control of TCP connections, in: Proceedings of 42nd Annual Conference on Information Sciences and Systems, pp. 1195-1200. 
[17] J. Cao, C. Nyberg, Linear programming relaxations and marginal productivity index policies for the buffer sharing problem, Queueing Systems 60 (2008) 247-269.

[18] P. Jacko, Dynamic Priority Allocation in Restless Bandit Models, Lambert Academic Publishing, 2010.

[19] C. Courcoubetis, R. Weber, Pricing Communication Networks: Economics, Technology and Modelling, John Wiley \& Sons Ltd, Chichester, England, 2003.

[20] R. Srikant, The Mathematics of Internet Congestion Control, Birkhäuser, Boston, Cambridge, Massachusetts, 2004.

[21] M. Lestas, A. Pitsillides, P. Ioannou, Congestion control in computer networks, in: Modeling and Control of Complex Systems, CRC Press, 2008.

[22] S.H. Low, R. Srikant, A mathematical framework for designing a low-loss, low-delay Internet, Networks and Spatial Economics 4 (2004) $75-101$.

[23] S.H. Low, F. Paganini, J.C. Doyle, Internet congestion control, IEEE Control Systems Magazine 22 (2002) 28-43.

[24] S.H. Low, D.E. Lapsley, Optimization flow control-I: basic algorithm and convergence, IEEE/ACM Transactions on Networking 7 (1999) $861-874$

[25] J. Niño-Mora, Dynamic priority allocation via restless bandit marginal productivity indices, TOP 15 (2007) 161-198.

[26] P.J. Brockwell, J. Gani, S.I. Resnick, Birth, immigration and catastrophe processes, Advances in Applied Probability 14 (1982) 709-731

[27] J. Niño-Mora, Restless bandits, partial conservation laws and indexability, Advances in Applied Probability 33 (2001) 76-98.

[28] R. Weber, Comments on: dynamic priority allocation via restless bandit marginal productivity indices, TOP 15 (2007) 211-216.

[29] M. Handley, C. Raiciu, D. Wischik, Restless bandits and congestion control, in: Newton Institute Workshop on New Topics at the Interface Between Probability and Communications, 2010. Http://www.cs.ucl.ac.uk/staff/d.wischik/Talks/dp.html.

[30] P. Jacko, B. Sansò, Congestion avoidance with future-path information, in: Proceedings of the EuroFGI Workshop on IP QoS and Traffic Control, IST Press, 2007, pp. 153-160.

[31] S. Qu, J.C. Gittins, A forwards induction approach to candidate drug selection, Advances in Applied Probability 43 (3) (2011) 649-665.

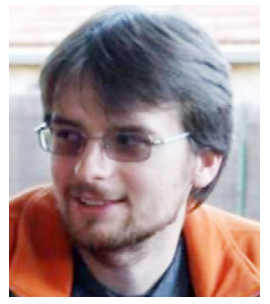

Peter Jacko is currently with the BCAM-Basque Center for Applied Mathematics, Spain. He obtained his Ph.D. in Business Administration and Quantitative Methods (2009) and D.E.A. in Statistics and Operations Research (2006) from the Universidad Carlos III de Madrid, Spain. He obtained his Mgr. (2003) and Bc. (2002) degrees in Mathematics from the Univerzita P.J. Šafárika v Košiciach, Slovakia.

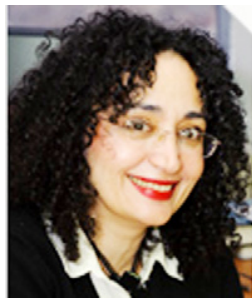

Brunilde Sansò is a full professor of electrical engineering at Ecole Polytechnique de Montreal and director of the LORLAB. Her interests are in performance, reliability, design, and optimization of wireless and wireline networks. She is a recipient of several awards, Associate Editor of Telecommunication Systems, and editor of two books on planning and performance. 